after injection (donor RBCs constituted 4 to $6 \%$ of the total number of RBCs at this time point). Results were independent of the dye used. Control experiments with unlabeled $\mathrm{CD} 47^{-1-} \mathrm{RBCs}$ in wild-type recipients and wild-type $\mathrm{RBCS}$ in $\mathrm{CD} 47^{-/-}$recipients (detection by staining for CD47 and flow cytometry) gave similar results.

18. N. van Rooijen and A. Sanders, J. Immunol. Methods 174, 83 (1994)

19. N. van Rooijen, N. Kors, G. Kraal, J. Leukocyte Biol. 45 97 (1989).

20. Dichloromethylene diphosphonate (clodronate; a gift from Roche Diagnostics/Boehringer Mannheim $\mathrm{GmbH}$ ) liposomes was prepared as described (18). Clodronate $(200 \mu \mathrm{l})$ or $\mathrm{NaCl}$ liposomes were given to recipient mice by tail-vein injection at 48 and 24 hours before RBC transfer. Immunohistochemical analyses revealed a complete macrophage depletion in recipient spleens 48 hours after a single injection of clodronate liposomes.

21. Spleen specimens were taken from recipients at 20 hours after the administration of PKH26-stained CD $47^{-1-}$ RBCs. Spleens were embedded in O.C.T compound (Sakura Finetek U.S.A., Torrance, CA), frozen in liquid nitrogen, and stored at $-80^{\circ} \mathrm{C}$. Cryostat sections $(8 \mu \mathrm{m})$ were incubated with antibodies to F4/80 [red pulp macrophages (34)] or MOMA-1 [marginal metallophilic macrophages (35)]. After incubation with horseradish peroxidase (HRP)-conjugated secondary antibodies, $\mathrm{F} 4 / 80^{+}$and $\mathrm{MOMA}-1^{+}$cells were visualized with Tyramide Signal Amplification (NEN Life Science Products, Boston, MA).

22. Y. Fujioka et al, Mol. Cell. Biol. 16, 6887 (1996).

23. S. Adams et al, J. Immunol. 161, 1853 (1998).

24. Wild-type spleens were digested with collagenase $D$ (Roche Diagnostics, Indianapolis, IN) $[400 \mathrm{U} / \mathrm{ml}$ in Hanks balanced salt solution (HBSS) without $\mathrm{Ca}^{2+}$ or $\mathrm{Mg}^{2+}$ ] for $30 \mathrm{~min}$ at $37^{\circ} \mathrm{C}$. After RBC lysis and extensive washing, the cells were resuspended to $5 \times$ $10^{7} / \mathrm{ml}$ in ice-cold HBSS with $1 \mathrm{mM} \mathrm{Ca}^{2+}, 1 \mathrm{mM}$ $\mathrm{Mg}^{2+}, 1 \%$ human serum albumin (HSA). Macrophages were allowed to adhere for $60 \mathrm{~min}$ at $37^{\circ} \mathrm{C}$ in plastic eight-well Lab-Tek chamber slides $(200 \mu \mathrm{l} /$ well; Nalgene Nunc International, Naperville, IL). Anti-SIRP $\alpha$ P84 (5 $\mu$ g) (9) or the isotype-matched control (anti-murine CD14; clone rmC5-3; Pharmingen) or no antibody was present during the last $15 \mathrm{~min}$. Subsequently, $50 \mu \mathrm{l}$ of PKH26-labeled wild-type or CD47-1- RBCs were added to each well for another $60 \mathrm{~min}$. Nonadherent cells were washed off with warm phosphate-buffered saline (PBS) $-0.1 \%$ HSA and noningested RBCs were lysed with ACK lysis buffer. Phagocytosis index (the number of RBCs phagocytosed per 100 adherent macrophages) was determined by fluorescence microscopy (Zeiss Axioscope).

25. A. Veillette, E. Thibaudeau, S. Latour, J. Biol. Chem. 273, 22719 (1998).

26. W. H. Fridman, P. Bruhns, O. Malbec, M. Daëron, J. Biol. Chem. 274, 32493 (1999).

27. Bone marrow-derived macrophages were prepared as described (36). Mature macrophages $\left(1.5 \times 10^{6}\right)$ were plated on $60-\mathrm{mm}$ bacteriological plastic Petri dishes (Valmark; Midwest Scientific, St. Louis, MO) for 16 hours before experiments. The cells were rinsed once with PBS, and $2 \mathrm{ml}$ of cold, serum-free Iscove's modified DMEM (IMDM) was added to each dish. After $2 \mathrm{~min}$ at $4^{\circ} \mathrm{C}, 2 \times 10^{6}$ wild-type or CD47 ${ }^{-1-}$ RBCs were added, then allowed to settle for $3 \mathrm{~min}$ at $4^{\circ} \mathrm{C}$, after which sodium pervanadate (Sigma) was added to $2 \mathrm{mM}$ and cells were transferred to a water bath at $37^{\circ} \mathrm{C}$. At the times indicated, the medium was quickly aspirated, and cells were lysed in cold lysis buffer [50 mM tris- $\mathrm{HCl}(\mathrm{pH} 7.5)$, $150 \mathrm{mM} \mathrm{NaCl}, 1 \% \mathrm{NP}-40$ (Pierce, Rockford, IL), 1 mM diisopropyl, aprotinin $(10 \mu \mathrm{g} / \mathrm{ml}), 2 \mathrm{mM}$ sodium pervanadate]. Lysates were cleared by centrifugation and incubated for 2 hours at $4^{\circ} \mathrm{C}$ with anti-SIRP monoclonal antibody (mAb) P84 bound to $20 \mu \mathrm{l}$ of goat anti-rat Sepharose (ICN, Costa Mesa, CA) for 2 hours at $4^{\circ} \mathrm{C}$. After two washes in lysis buffer, immunoprecipitated proteins were eluted with reducing SDS-polyacrylamide gel electrophoresis (PAGE) sample buffer, separated on $8 \%$ SDS-PAGE, and transferred to a polyvinylidene difluoride membrane (Millipore, Bedford, MA). Tyrosine phosphorylation was detected by Western blot with anti-phosphotyrosine mAb 4G10, followed by HRP-conjugated secondary antibody and enhanced chemoluminescence as described (36).

28. Reviewed in N. D. Avent and M. E. Reid, Blood 95, 375 (2000)

29. F. P. Lindberg et al., unpublished data.

30. L. G. Poels et al., J. Natl. Cancer Inst. 76, 781 (1986).

31. M. Seiffert et al, Blood 94, 3633 (1999).

32. P. Mombaerts et al., Cell 68, 869 (1992).

33. M. R. Wessels et al., Proc. Natl. Acad. Sci. U.S.A. 92 11490 (1995).

34. M. D. Witmer and R. M. Steinman, Am. J. Anat. 170 465 (1984)

35. G. Kraal and M. Janse, Immunology 58, 665 (1986).
36. T. Roach et al., Curr. Biol. 7, 408 (1997).

37. We thank T. Steinberg, W. Yokoyama, J. Atkinson, and E. Brown for critically reading the manuscript; $D$. Chaplin, H. Molina, and P. Leenen for reagents; and E. Ford for technical assistance. Supported by grants from the NIH (GM57573-01), the American Diabetes Association, the Washington University-Monsanto Research Agreement, a pilot grant from the Howard Hughes Medical Institute, and the Medical Research Service of the Depatment of Veterans Affairs. P.-A.O is supported by a postdoctoral fellowship from the Umeå University-Washington University exchange program and by the Swedish Medical Research Council.

15 February 2000; accepted 25 April 2000

\title{
Selectivity for 3D Shape That Reveals Distinct Areas Within Macaque Inferior Temporal Cortex
}

\author{
Peter Janssen, Rufin Vogels, * Guy A. Orban
}

\begin{abstract}
The anterior part of the macaque inferior temporal cortex, area TE, occupies a large portion of the temporal lobe and is critical for object recognition. Thus far, no relation between anatomical subdivisions of TE and neuronal selectivity has been described. Here, we present evidence that neurons selective for three-dimensional (3D) shape are concentrated in the lower bank of the superior temporal sulcus, whereas neurons in lateral TE are generally unselective for 3D shape, though equally selective for 2D shape. These findings reveal that TE consists of at least two distinct areas, one of which processes a specific object property.
\end{abstract}

The inferior temporal cortex (IT) is part of the ventral visual stream, which is known to be critical for object recognition $(1,2)$. Neurons in the anterior part of IT, area TE (Fig. 1), respond selectively to object attributes (3). For over three decades, researchers have been puzzled by the organization of this large cortical region [up to $380 \mathrm{~mm}^{2}$ (4)]. Although TE can be divided into a number of subregions on the basis of anatomical criteria (5), no clear link between the anatomy and neuronal selectivity has yet emerged. Here, we present evidence, based on 3D shape-selectivity, that at least two areas can be distinguished within TE: the lower bank of the superior temporal sulcus (STS) and lateral TE.

Thirty-two pairs of disparity-defined (6) curved 3D shapes served as stimuli. The 3D shape-selectivity was assessed by comparing the responses of single TE neurons to the members of pairs of 3D shapes differing only in the sign of their binocular disparity (concave versus convex) (7). Reconstruction of

Laboratorium voor Neuro-en Psychofysiologie, Medical School, Katholieke Universiteit Leuven, Leuven B-3000, Belgium.

*To whom correspondence should be addressed. Email: Rufin.Vogels@med.kuleuven.ac.be the position of each recorded neuron (Fig. 1) (8) showed that 142 neurons were recorded in the lower bank of the STS and 82 in lateral TE (9). Figure 2A illustrates the responses of an STS neuron that responded strongly to the concave, but not to the convex shape (10). Monocular presentations to left or right eye separately evoked only minimal responses (11). This 3D shape-selective response contrasted markedly with the responses of lateral TE neurons. Although the lateral TE neuron of Fig. 2B responded to the concave shape, the response to the convex shape was equally strong. Moreover, the monocular presentations elicited responses that were similar to those in the stereo conditions. This response pattern, combined with the 2D shape-selectivity revealed by the search test (7), indicates that this neuron was not sensitive to $3 \mathrm{D}$ structure, but simply responded to the $2 \mathrm{D}$ shape.

Overall, $56 \%$ of the tested STS neurons were selective for 3D shape, compared to only $12 \%$ in lateral TE $\left(\chi^{2}=40.9, P<\right.$ $0.001)(12,13)$. Moreover, the degree of $3 \mathrm{D}$ selectivity was much lower in lateral TE (14), though the mean net response to the preferred 3D shape did not differ significantly between the two subdivisions (20.2 and 19.1 spikes per second for the STS and lateral TE, re- 


\section{R E P O R T S}

spectively. Kolmogorov-Smirnov test, nonsignificant). The difference in the population response to preferred and nonpreferred 3D shape is larger for STS than for lateral TE neurons (Fig. 3A) (15). Differences in population responses contain an analysis-induced component because the stimulus evoking the largest response is always selected as the preferred stimulus, even for nonsignificant response differences. The population of lateral TE neurons was no more sensitive to 3D structure than a population of statistically nonselective neurons ( $n=135$, Fig. 3A), derived from STS (47\%) and lateral TE $(53 \%)$.

We tested whether 3D shape-selective neurons respond to particular disparity values within the stimulus (far versus near), or, alternatively, to spatial variations of disparity (concave versus convex) by presenting the preferred and nonpreferred 3D shapes at five different positions in depth. The large majority of STS neurons tested (37/42) responded to spatial variations of disparity, not to local disparity values. None of four lateral TE neurons tested could be classified as responsive to disparity variations.

The difference in 3D shape-selectivity between STS and lateral TE might reflect a mere generalized difference in stimulus selectivity for the stimuli we used. However, lateral TE neurons were as selective for 2D shape as the neurons in the STS (16), which indicates that the difference between STS and lateral TE was indeed due to 3D shapeselectivity. Although lateral TE neurons are clearly selective for 2D shape, they are generally not selective for $3 \mathrm{D}$ shape, even for large disparity values (17).

The response of the STS neurons to the preferred 3D shape was generally larger than the sum of the monocular responses (Figs. 2A and $3 \mathrm{~B}$ ). Typically, the opposite was true for lateral TE neurons (Figs. 2B and 3B). We defined a binocular summation index as (response to preferred $3 \mathrm{D}$ shape) - (sum of the monocular responses)/(sum of the monocular responses). The median binocular summation index for the STS neurons $(+0.18 ; n=142)$ was significantly larger than that for the lateral TE neurons $(-0.41 ; n=82)$ (18). The binocular summation observed in STS neurons reflects their selectivity for 3D shape, because binocular presentation of the nonpreferred 3D shape, which contained the same monocular images as the preferred 3D shape (Fig. 2A), evoked no binocular summation (median summation index $=-0.65$ ).

The rostral part of the lower bank of the
Fig. 1. Anatomical reconstruction of the recording sites. (A) Superposition of the MR (blue) and the CT coronal section (black). The vertical bar is the guiding tube, the red line the electrode track. (B) Lateral view of the macaque brain showing the anterior-posterior extent of the recordings (vertical lines). The shaded area corresponds to area TE. (C) Estimated recording zones in STS (blue) and lateral TE (green). The red lines indicate the medio-lateral range used in (D), the right one corresponds to the penetration shown in (B). Arrows indicate fundus (a) and lip (b) of the STS, and lateral border of the anterior middle temporal sulcus (AMTS) (c). (D) Average depth (in micrometers) of the silent zones (dotted lines) and the skull (full line), for three medio-lateral bins spaced $2 \mathrm{~mm}$ apart. Same scale as in (C). (E) Flattened map of the estimated recording area (average of the two monkeys). (a), (b), and (c) are as in (C). The pale blue
Fig. 2. Responses of STS (A) and lateral TE neurons (B). The left panel illustrates the images presented to left and right eye for the preferred (top row) and the nonpreferred (bottom row) 3D shape. The middle panel illustrates the perceived 3D structure (semi-cylindrically concave and convex). This illustration does not display the actual borders of the stimulus. The right panel shows the neuronal responses to binocular ("stereo") and monocular ("left eye" and "right eye") presentation of preferred and nonpreferred 3D shapes. Bin width is $20 \mathrm{~ms}$.
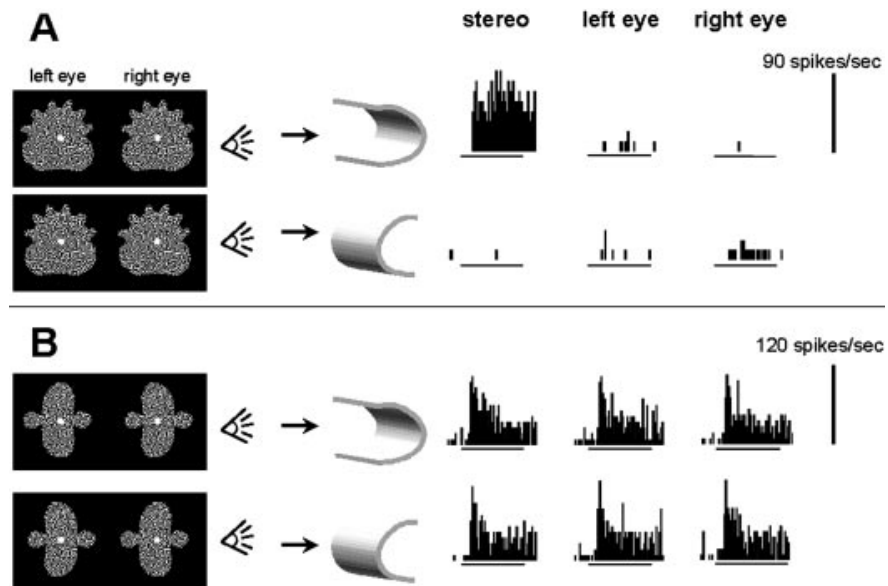

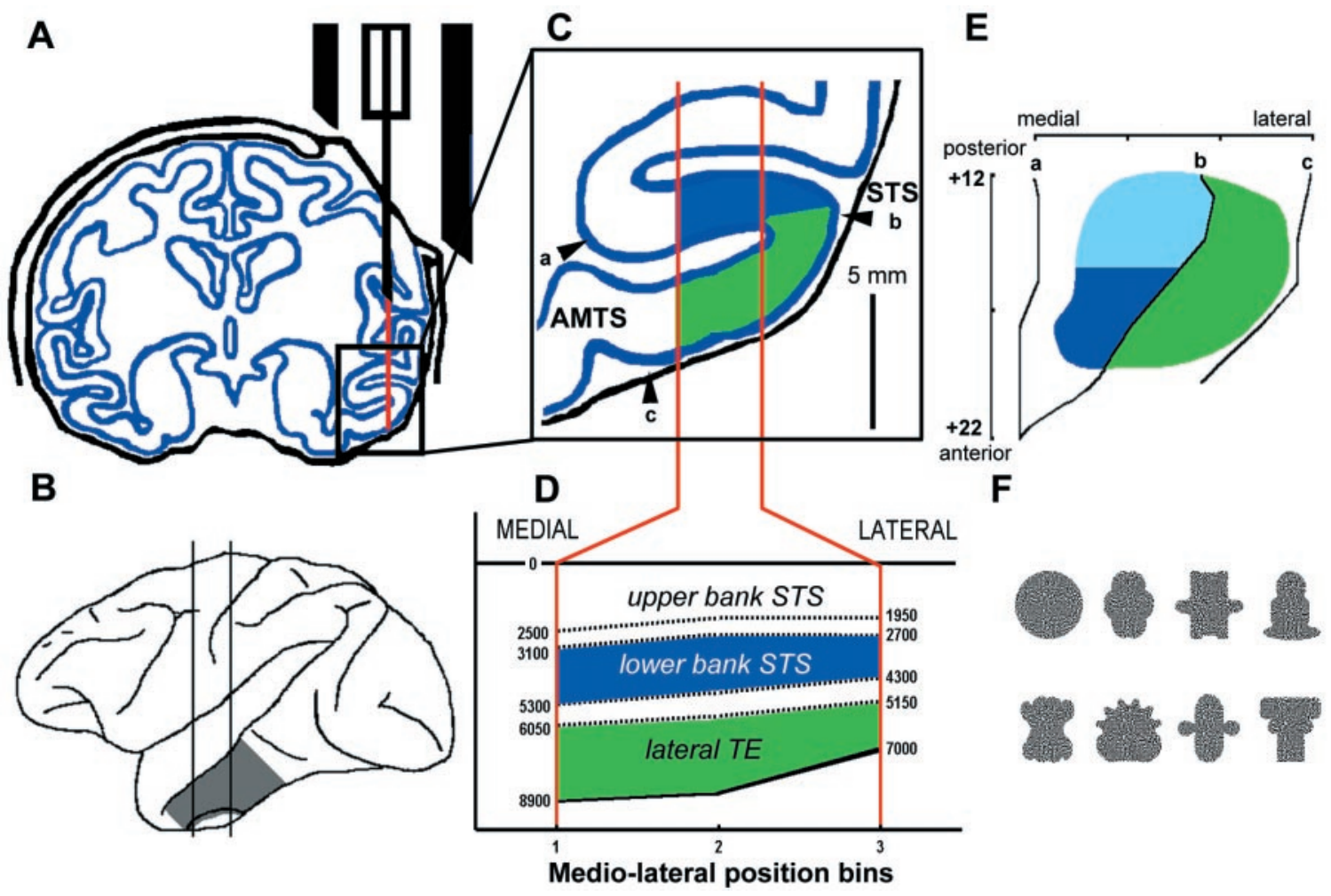

area indicates that posteriorly, STS neurons were weakly responsive. Most responsive neurons were recorded in rostral STS. (F) Shown are the 2D shapes used to derive the 3D stimuli. 
A Superior Temporal Sulcus Lateral TE Nonselective neurons
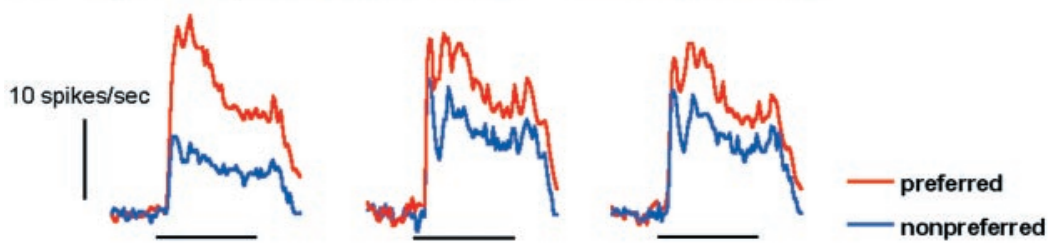

B
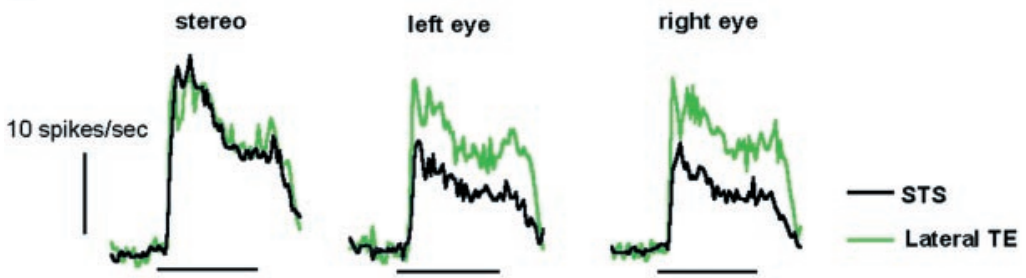

Fig. 3. Population responses. (A) Population peristimulus-time histograms (PSTHs) for preferred (red) and nonpreferred (blue) 3D shape, for STS (left panel), lateral TE (middle panel), and all statistically nonselective neurons (right panel). (B) Population PSTHs for the response to the preferred 3D shape in the stereo and in the monocular conditions, for STS (black) and lateral TE neurons (green).

STS corresponds to cytoarchitectonic areas TEa and TEm (5). The connectivity of this region is distinct from that of lateral TE (9). The cortex in the intraparietal sulcus (IPS), the terminus of the dorsal visual pathway, connects with the lower bank of the STS, but less so with lateral TE $(19,20)$. In addition, the lower STS is more closely connected to TEav than to TEad (21). Our results suggest a specific role for this pivotal brain region: the lower bank of the STS is the part of TE which processes disparity-defined 3D shape $(22,23)$. This finding has two major implications. Firstly, our results provide a functional role for the parietal connections of the STS, because the caudal bank of the IPS contains neurons selective for disparity-defined orientation in depth (24). Thus, these two interconnected areas, belonging to the dorsal and to the ventral streams, are both involved in the processing of 3D-structure (25). Secondly, in view of the distinct cyto- and myeloarchitecture, the specific pattern of connections and the functional specialization of the lower bank of the STS, this region should be regarded as a distinct area within TE.

\section{References and Notes}

1. L. G. Ungerleider and M. Mishkin, in Analysis of Visual Behavior, D. J. Ingle, M. A. Goodall, R. J. Mansfield, Eds. (MIT Press, Cambridge, MA, 1982).

2. N. K. Logothetis and D. L. Sheinberg, Annu. Rev. Neurosci. 19, 577 (1996).

3. K. Tanaka, H. K. Fukuda, Y. Moriya, J. Neurophysiol. 66, 170 (1991).

4. I. Fujita, K. Tanaka, M. Ito, K. Cheng, Nature 360, 343 (1992).

5. B. Seltzer and D. N. Pandya, Brain Res. 149, 1 (1978).

6. Binocular disparity is a powerful depth cue.

7. We imposed four pairs of depth profiles onto each of eight simple 2D shapes (Fig. 1F). The members of a pair of 3D shapes use the same two monocular images [P. Janssen, R. Vogels, G. A. Orban, Proc. Natl. Acad. Sci.
U.S.A. 96, 8217 (1999)]. The 32 stimuli in our search test (one member of each 3D pair) differed in 2D shape as well as in 3D structure. Neurons showing differential activity in the search test were tested by presenting the members of two 3D pairs, all derived from a single 2D shape. The stimuli were presented dichoptically by means of liquid crystal shutters (60 Hz per eye). Stimulus luminance and contrast was $2.5 \mathrm{~cd} / \mathrm{m}^{2}$ and $4(\Delta / / I)$, respectively. The maximal stimulus diameter was 5.5 degrees, dot density $50 \%$ and dot size 4 arcmin. A fixation target (24 arcmin) was superimposed on the stimulus. The fixation distance was $86 \mathrm{~cm}$ and the maximal amplitude of the disparity gradient was 0.65 degrees. Stimulus duration was $800 \mathrm{~ms}$. Presentation of shapes filled with three different textures was interleaved. Two emmetropic rhesus monkeys were trained to fixate within 0.7 degree of a small target. The position of the right eye was recorded with a scleral search coil. Surgical procedures and animal treatment were in accordance with the guidelines established by the $\mathrm{Na}$ tional Institutes of Health (NIH) for the care and use of laboratory animals.

8. For each vertical penetration, we noted the depths of responsive neurons, and of landmarks including white to gray matter transitions and the skull. A computer tomography (CT) scan obtained with the guiding tube in situ confirmed that the recording chambers were implanted at the targeted coordinates ( $16 \mathrm{~mm}$ anterior, 22 $\mathrm{mm}$ lateral). To reconstruct the recording positions, the $C T$ and the magnetic resonance (MR) images were superimposed (Fig. 1, A and C). In Fig. 1D, all penetrations except the most lateral ones (in which no transition between STS and lateral TE was detected) were classified into three bins according to their mediolateral position, and the landmark depths were averaged for each bin separately. For the most lateral penetrations, we classified the neurons recorded in the upper $1000 \mu$ as belonging to the lower bank of the STS, and all other neurons as lateral TE neurons. Excluding neurons recorded in this transition zone $(n=40)$ did not alter the results. The significance $(P<0.05)$ of the 3D shape-selectivity was tested by analysis of variance (ANOVA) on the net responses (interval $=400 \mathrm{~ms}$ ). The 3D shape-selectivity was deemed not to arise from purely monocular mechanisms if the difference in response between the dichoptic presentations was at least three times greater than the difference in the sum of the monocular responses.

9. This region corresponds to TEad [K. S. Saleem and K. Tanaka, J. Neurosci. 16, 4757 (1996)].

10. ANOVA: $F(3,20)=41.3, P<0.0001$.

11 . For every responsive neuron, the horizontal right eye position was analyzed in the same intervals as the neural activity. This analysis showed only small (less than 3 arcmin) eye deviations during stimulus presentation. Experiments with coils implanted in both eyes confirmed that no vergence eye movements were made upon stimulus presentation.

12. For monkey J., the proportion of 3D shape-selective neurons equaled $61 \%$ in the STS and $12 \%$ in lateral TE $\left(\chi^{2}=30.32, P<0.01\right)$. For monkey $L$, these proportions equaled $44 \%$ in the STS and $12 \%$ in lateral TE $\left(\chi^{2}=6.5, P<0.01\right)$.

13. In both monkeys, the total extent of cortex covered measured at least $6 \mathrm{~mm}$ in the anterior-posterior direction, and $5 \mathrm{~mm}$ in the medio-lateral direction (Fig. 1E). We made 139 penetrations (68 in lateral TE and 71 in the STS) and recorded a total of 828 neurons, 604 of which were unresponsive. Because 3D shape-selective neurons were uniformly distributed in STS, it is highly unlikely that we missed clusters of such cells in lateral TE.

14. The median 3D-shape selectivity index [(response to the preferred 3D shape) - (response to other member of the pair)/(response to preferred 3D shape)] equaled 0.82 (1st quartile: 0.66 ; 3rd quartile: $0.97 ; n=79$ ) for the 3D shape-selective STS neurons, compared to 0.58 for the selective neurons in the lateral TE (1st quartile: 0.35 ; 3rd quartile: $1.0 ; n=10$ ).

15. Non-normalized population peristimulus-time histograms (PSTHs) were computed by averaging the net activity for each population of neurons (STS, lateral TE and nonselective neurons). The population response to the nonpreferred 3D shape was subtracted binwise from the response to the preferred 3D shape. The ANOVA was computed on the resulting differential population responses. The difference between STS and lateral TE neurons proved significant $[F(1,34)=155.9, P<0.0001]$.

16. The 2D-shape index [G. Rainer, W. F. Assaad, E. K. Miller, Nature 393, 577 (1998)] was defined as: ( $n-$ $\left.\Sigma r_{\mathrm{i}} / \max \right) /(n-1)$ where $n$ is the number of $2 \mathrm{D}$ shapes, $r_{\mathrm{i}}$ is the mean net response to each 2D shape, and $\max$ is the largest mean response. For every $2 \mathrm{D}$ shape, we averaged the responses to the four different 3D shapes derived from that 2D shape. The average index was nearly identical in STS (0.66) and lateral TE (0.65).

17. The proportion of 3D shape-selective neurons was very similar $(10 \%)$ in a population of 76 lateral TE neurons tested with larger disparity amplitudes (1.3 degrees).

18. Kolmogorov-Smirnov two-sample test $P<0.001$. For negative monocular responses, the index was set to +10 ( $7 \%$ of the neurons).

19. See figure 12 in J. S. Baizer, L. G. Ungerleider, R. Desimone, J. Neurosci. 11, 168 (1991).

20. E. G. Jones and T. P. S. Powell, Brain 93, 793 (1970).

21. K. S. Saleem, W. Suzuki, K. Tanaka, T. Hashikawa. J. Neurosci., in press.

22. Lateral TE neurons may be sensitive to 3D structure defined by monocular depth cues, such as shading [M. Ito, I. Fujita, H. Tamura, K. Tanaka, Cerebr. Cortex 4, 499 (1994)].

23. G. Baylis, E. T. Rolls, and C. M. Leonard [J. Neurosci. 7, 330 (1987)] reported that STS neurons were more selective and more frequently responsive to faces and complex stimuli than neurons in lateral TE. Face neurons, however, can be found throughout TE [R. Desimone, J. Cogn. Neurosci. 3, 1 (1991)]. Using stimulus dimensions other than 3D shape may reveal additional differences between STS and lateral TE.

24. E. Shikata, Y. Tanaka, H. Nakamura, M. Taira, H. Sakata, Neuroreport 7, 2389 (1996).

25. Three-dimensional information could also reach the STS through area FST [B. Seltzer and D. N. Pandya, J. Comp. Neurol. 290, 451 (1989)], which receives projections from area MT/V5 [L. G. Ungerleider and R. Desimone, J. Comp. Neurol. 248, 190 (1986)].

26. Supported by Geneeskundige Stichting Koningin Elisabeth, FWO Vlaanderen, GOA 95-99/06 and GOA 2000/11. P.J. is a research assistant and R.V. is a research associate of the FWO. We thank M. Depaep, P. Kayenbergh, G. Meulemans, G. Vanparrys, and W. Spileers.

2 February 2000; accepted 28 April 2000 